\title{
Singularity Structure of the Two-Point Function in Quantum Field Theory in Curved Spacetime*
}

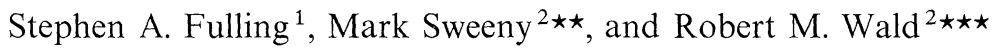 \\ ${ }^{1}$ Department of Mathematics, Texas A\&M University, College Station, Texas 77843, USA \\ 2 Enrico Fermi Institute, University of Chicago, Chicago, Illinois 60637, USA
}

\begin{abstract}
In the point-splitting prescription for renormalizing the stress-energy tensor of a scalar field in curved spacetime, it is assumed that the anticommutator expectation value $G\left(x, x^{\prime}\right)=\left\langle\phi(x) \phi\left(x^{\prime}\right)+\phi\left(x^{\prime}\right) \phi(x)\right\rangle$ has a singularity of the Hadamard form as $x \rightarrow x^{\prime}$. We prove here that if $G\left(x, x^{\prime}\right)$ has the Hadamard singularity structure in an open neighborhood of a Cauchy surface, then it does so everywhere, i.e., Cauchy evolution preserves the Hadamard singularity structure. In particular, in a spacetime which is flat below a Cauchy surface, for the "in" vacuum state $G\left(x, x^{\prime}\right)$ is of the Hadamard form everywhere, and thus the point-splitting prescription in this case has been rigorously shown to give meaningful, finite answers.
\end{abstract}

A great deal of attention has been focused recently on the problem of renormalizing the stress energy tensor, $T_{\mu v}$, of a quantum field in curved spacetime. The quantum field operator itself is mathematically well defined as a distribution, but $T_{\mu \nu}$ is formally a product of field operators (i.e., a product of distributions) and hence is ill-defined until further rules are given for how to evaluate it. The evaluation of $T_{\mu \nu}$ is of considerable interest since it governs the back reaction of the quantum field upon the spacetime geometry.

One procedure for evaluating $T_{\mu \nu}$ which has been studied in detail is the pointsplitting renormalization method [1-7]. The basic idea of this approach is to regard $T_{\mu \nu}$ initially as a two-point operator-valued distribution, $\mathscr{T}_{\mu \nu}\left(x, x^{\prime}\right)$. $\mathscr{T}_{\mu \nu}\left(x, x^{\prime}\right)$ is well defined, but the "coincidence limit" $x \rightarrow x^{\prime}$ is singular. As described below, it has been assumed that the singularity structure of $\mathscr{T}_{\mu v}\left(x, x^{\prime}\right)$ as $x \rightarrow x^{\prime}$ is of a certain local form. (This has been verified in special cases and there are formal arguments suggesting that it holds in general.) The renormalization ansatz is to subtract from $\mathscr{T}_{\mu \nu}\left(x, x^{\prime}\right)$ another, locally constructed, distribution which has the

* Supported in part by NSF grants PHY 77-01432 to Texas A\&M University and PHY 76-81102 to the University of Chicago, and by the Alfred P. Sloan Foundation

$\star \star$ NSF Predoctoral Fellow

$\star \star \star$ Sloan Foundation Fellow 
same singularity structure as $x \rightarrow x^{\prime}$. The renormalized value of $T_{\mu \nu}(x)$ is then defined as the coincidence limit of this difference.

To make these ideas more precise, consider the case of a real, massless, KleinGordon scalar field $\phi$. Analogous results to those discussed here apply to other fields. Rather than work with $\mathscr{T}_{\mu \nu}\left(x, x^{\prime}\right)$ it is easier to work with the anticommutator function $G\left(x, x^{\prime}\right)$ defined by

$$
G\left(x, x^{\prime}\right)=\left\langle\phi(x) \phi\left(x^{\prime}\right)+\phi\left(x^{\prime}\right) \phi(x)\right\rangle
$$

where the expectation value is taken in the state of interest (e.g., the "in" or "out" vacuum). The expectation value of $\mathscr{T}_{\mu \nu}\left(x, x^{\prime}\right)$ can be generated from $G\left(x, x^{\prime}\right)$ by differentiation. The starting point of the point-splitting prescription is the assumption that $G\left(x, x^{\prime}\right)$ - which initially is known only to be a two-point distribution - can actually be realized as the principal part of a function with singularity structure of the form

$$
G\left(x, x^{\prime}\right)=\frac{u\left(x, x^{\prime}\right)}{\sigma}+v\left(x, x^{\prime}\right) \ln \sigma+w\left(x, x^{\prime}\right) .
$$

Here $\sigma$ is half the square of the geodesic distance between $x$ and $x^{\prime}$ (taken to be positive for spacelike separations); $u, v$, and $w$ are smooth functions with $v$ and $w$ expandable as

$$
v\left(x, x^{\prime}\right)=\sum_{n} v_{n}\left(x, x^{\prime}\right) \sigma^{n}, \quad w\left(x, x^{\prime}\right)=\sum_{n} w_{n}\left(x, x^{\prime}\right) \sigma^{n}
$$

with $u, v_{n}$, and $w_{n}$ satisfying the Hadamard recursion relations [8-10]. The Hadamard recursion relations uniquely determine $u\left(x, x^{\prime}\right)$ and $v_{n}\left(x, x^{\prime}\right)$ in a local manner [10] (determined by the metric and its derivatives on the geodesic connecting $x$ and $x^{\prime}$ ). The series in (3) can be shown to have a finite radius of convergence in an analytic spacetime [10]; in the $C^{\infty}$, non-analytic case we require only that these series be asymptotic around $\sigma=0$. The right-hand side of (2) is well defined at most for $x$ within a normal neighborhood [11] of $x^{\prime}$, for if more than one geodesic (or no geodesics at all) connect $x$ with $x^{\prime}$, then even $\sigma$ is ill defined. Thus (2) is supposed to hold only within a normal neighborhood. This latter point does not cause difficulties in the renormalization prescription for $T_{\mu \nu}$ since only the behavior of $G\left(x, x^{\prime}\right)$ in the coincidence limit is required.

Solutions of the form given by the right-hand side of (2) were originally constructed by Hadamard in his study of second order, linear partial differential equations. We will refer to solutions of this form as Hadamard elementary solutions.

In [7] it was shown that if (2) is valid, then by subtracting a suitably constructed Hadamard solution from $G\left(x, x^{\prime}\right)$ one can define a point-splitting renormalization prescription for $T_{\mu \nu}$ that satisfies the requirements of axioms (1)-(4) of [6]. Since these axioms uniquely determine a renormalized stress energy operator up to the addition of conserved local curvature tensors, this solves the stress-energy renormalization problem modulo the remaining ambiguity of addition of these curvature terms. However, this result rests on the validity of (2). If (2) is not valid, then the point-splitting prescriptions that have been studied thus far would not yield finite answers. Since the other renormalization prescriptions 
are mathematically very closely related to point-splitting, it is likely that violation of (2) would also signal similar difficulties in these approaches.

In this paper, we study the validity of (2). We prove that if (2) holds in a neighborhood of a Cauchy surface, then it is valid everywhere in the spacetime. While this does not prove the general validity of (2) for all physically reasonable states, it points strongly in that direction. In particular, if we consider the mathematically simple example of a globally hyperbolic spacetime which is flat to the past of a Cauchy surface (i.e., a gravitational field which is "turned on" after a finite time), then $G\left(x, x^{\prime}\right)$ for, say, the "in" vacuum state will take in the past the flat space value, which is known to be of the Hadamard form [12]. Our results prove that whatever spacetime curvature may be present in the future, $G\left(x, x^{\prime}\right)$ remains of the Hadamard form and thus the point-splitting renormalization prescription will give meaningful, finite answers for $\left\langle 0_{\text {in }}\left|T_{\mu \nu}\right| 0_{\text {in }}\right\rangle$ throughout the spacetime. For general spacetimes, our results show that the infrared divergences considered by Ford and Parker [13] cannot arise dynamically from a state where $G\left(x, x^{\prime}\right)$ initially is of the Hadamard form.

All these considerations suggest that the validity of (2) be regarded as a basic criterion for a "physically reasonable" state, perhaps even as the definition of that phrase. This raises the possibility of constructing quantum states from two-point distribution solutions of the field equation by a procedure of the Wightman or GNS type [14], bypassing the quantization of normal modes in a Fock space.

The precise theorem we shall prove is the following:

Theorem. Let $\left(M, g_{\mu v}\right)$ be a $C^{\infty}$, globally hyperbolic spacetime. Let $G\left(x, x^{\prime}\right)$ be a twopoint distribution on spacetime (defined on $C^{\infty}$ test functions of compact support) which satisfies the (curved space) wave equation in both $x$ and $x^{\prime}$. Suppose that in an open neighborhood $O$ of a Cauchy surface $S, G$ is of the Hadamard form, i.e., we can find a function having the form of the right-hand side of (2) such that for all test functions $f$ and $g$ with support in $\mathcal{O}$ (and both supports contained within a convex normal neighborhood) $G(f, g)$ is the principal value integral of this function "smeared" with $f$ and $g$. Then $G$ is of the Hadamard form throughout the spacetime.

Before proving this result, we should make some remarks about the Cauchy evolution of distributions. Let $D(x)$ be a distribution on spacetime, i.e., $D$ is a continuous linear map from $C^{\infty}$ test functions of compact support into $\mathbb{C}$. The statement that $D$ satisfies the wave equation, $\nabla^{\mu} \nabla_{\mu} D=0$, means that for all test functions $f$ of the form $f=\nabla^{\mu} \nabla_{\mu} h$ (where $h$ is a test function), we have $D(f)=0$. If $D$ satisfies the wave equation, then knowledge of it in an open neighborhood $\mathcal{O}$ of a Cauchy surface (i.e., on all test functions with support in $\mathcal{O}$ ) determines it everywhere. This is because every test function can be expressed as the sum of a test function having support in $\mathcal{O}$ with a test function of the form $\nabla^{\mu} \nabla_{\mu} h$. For example, for the test function $f$ illustrated in Fig. 1, we define $L$ to be the advanced solution of the wave equation with source $f$ and define $l=\alpha L$ where $\alpha$ is a $C^{\infty}$ function which is 1 outside $(\mathcal{O}$ to the future of $S$ and 0 outside $\mathcal{O}$ to the past of $S$. Then $l$ is a test function and

$$
g=\nabla^{\mu} \nabla_{\mu} l-f
$$




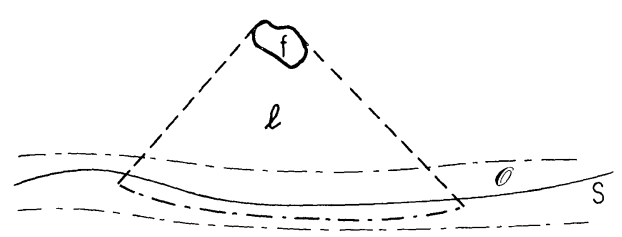

Fig. 1. The construction used to express $f$ as $\nabla^{\mu} \nabla_{\mu} l-g$

is a test function with support in $\mathcal{O}$. Linearity of $D$ implies

$$
D(f)=D\left(\nabla^{\mu} \nabla_{\mu} l\right)-D(g)=-D(g)
$$

thus showing that a knowledge of $D$ in $\mathcal{O}$ determines $D(f)$.

The basic idea of the proof of the theorem is to consider the difference of the distribution $G\left(x, x^{\prime}\right)$ and a symmetric, locally constructed Hadamard distribution $H\left(x, x^{\prime}\right)$ which satisfies the (inhomogeneous) wave equation in $x$ and $x^{\prime}$ with smooth source $J\left(x, x^{\prime}\right)$ :

$$
\begin{aligned}
& \square_{x} H\left(x, x^{\prime}\right)=J\left(x, x^{\prime}\right), \\
& \square_{x^{\prime}} H\left(x, x^{\prime}\right)=J\left(x^{\prime}, x\right) .
\end{aligned}
$$

(Below we shall show explicitly how to construct $H\left(x, x^{\prime}\right)$ and will deal with the technical problems which arise.) By hypothesis, the difference between $G$ and $H$ is a smooth function $F\left(x, x^{\prime}\right)$ in the open neighborhood $\mathcal{O}$ of the Cauchy surface $S$. We extend the definition of the smooth function $F$ by Cauchy evolution: Fixing $x^{\prime}$ in $\mathcal{O}$, we define $F\left(x, x^{\prime}\right)$ for all $x$ via its initial data on $S$ and the wave equation

$$
\square_{x} F\left(x, x^{\prime}\right)=-J\left(x, x^{\prime}\right) .
$$

Now, fixing $x$, we define $F\left(x, x^{\prime}\right)$ for all $x^{\prime}$ by its initial data on $S$ and the equation

$$
\square_{x^{\prime}} F\left(x, x^{\prime}\right)=-J\left(x^{\prime}, x\right) .
$$

Thus, by construction, $F$ satisfies (6) for all $x, x^{\prime} ; F$ also satisfies (5) for all $x, x^{\prime}$ since $I\left(x, x^{\prime}\right) \equiv \square_{x} F\left(x, x^{\prime}\right)+J\left(x, x^{\prime}\right)$ vanishes for $x^{\prime} \in \mathcal{O}$ and

$$
\begin{aligned}
\square_{x^{\prime}} I\left(x, x^{\prime}\right) & =\square_{x^{\prime}} \square_{x} F\left(x, x^{\prime}\right)+\square_{x^{\prime}} J\left(x, x^{\prime}\right) \\
& =\square_{x}\left(-J\left(x^{\prime}, x\right)\right)+\square_{x^{\prime}} J\left(x, x^{\prime}\right) \\
& =-\square_{x} \square_{x^{\prime}} H\left(x, x^{\prime}\right)+\square_{x^{\prime}} \square_{x} H\left(x, x^{\prime}\right) \\
& =0
\end{aligned}
$$

everywhere. Consequently, the distribution

$$
D\left(x, x^{\prime}\right)=F\left(x, x^{\prime}\right)-\left[G\left(x, x^{\prime}\right)-H\left(x, x^{\prime}\right)\right]
$$

satisfies the following two properties: (i) $D$ vanishes for $x, x^{\prime} \in \mathcal{O}$ (ii) $D$ everywhere satisfies the homogeneous wave equation in both $x$ and $x^{\prime}, \square \square_{x} D=\square_{x^{\prime}} D=0$. But the uniqueness argument on the Cauchy development of distributions given above proves that these properties imply $D=0$. Thus, we have everywhere

$$
G\left(x, x^{\prime}\right)=H\left(x, x^{\prime}\right)+F\left(x, x^{\prime}\right)
$$

where $F$ is a smooth function. This shows that $G$ is of the Hadamard form. 
To make the above argument into a complete proof of the theorem, all we need to do is construct the local Hadamard distribution $H\left(x, x^{\prime}\right)$ with the required properties. Unfortunately, some technical problems arise because (1) the series, (3), that one would like to write down to define $H$ may not converge, and (2) the quantities $\sigma, u$, and $v_{n}$ are, in any case, well defined only when $x$ lies in a normal neighborhood of $x^{\prime}$, and a careless attempt to extend their definition may violate the required smoothness of $H$. We overcome these problems by working with a Hadamard parametrix and by Cauchy evolving in "small steps" so that problems arising from the finite size of normal neighborhoods are avoided.

We construct our Hadamard parametrix $H\left(x, x^{\prime}\right)$ as follows: as mentioned above, the series

$$
v\left(x, x^{\prime}\right)=\sum_{n=0}^{\infty} v_{n}\left(x, x^{\prime}\right) \sigma^{n}
$$

is not guaranteed to converge. However, if we let $\psi: \mathbb{R} \rightarrow \mathbb{R}$ be a $C^{\infty}$ function which is 1 for $|x|<\frac{1}{2}$ and 0 for $|x|>1$, and if $\left\{\alpha_{n}\right\}$ is a sequence of real numbers which converges to 0 , then

$$
\tilde{v}\left(x, x^{\prime}\right)=\sum_{n=0}^{\infty} \psi\left(\sigma / \alpha_{n}\right) v_{n}\left(x, x^{\prime}\right) \sigma^{n}
$$

is guaranteed to converge, since either $\sigma=0$ and $\tilde{v}=v_{0}$ or $\sigma \neq 0$ and only finitely many terms contribute because of the cutoff by $\psi$. By making $\alpha_{n}$ converge to zero rapidly enough, we can ensure that $\tilde{v}$ is $C^{\infty}$ everywhere. Setting $\tilde{u}\left(x, x^{\prime}\right)$ $=\psi(\sigma / \beta) u\left(x, x^{\prime}\right)$, we define, for $x$ in some normal neighborhood of $x^{\prime}$,

$$
H\left(x, x^{\prime}\right)=\frac{\tilde{u}\left(x, x^{\prime}\right)}{\sigma}+\tilde{v}\left(x, x^{\prime}\right) \ln \sigma .
$$

Later we shall specify this neighborhood so that $H\left(x, x^{\prime}\right)$ can be set equal to zero at all $x$ outside the neighborhood at which we need to define $H$. (The cutoff factors in the parametrix will allow this to be done without violating the smoothness of $H$.) Since $u\left(x, x^{\prime}\right)$ and each $v_{n}\left(x, x^{\prime}\right)$ are symmetric ${ }^{1}$ in $x$ and $x^{\prime}$, so is $H\left(x, x^{\prime}\right)$. If we apply the wave operator in $x$ or $x^{\prime}$ to $H$, the resultant function $J\left(x, x^{\prime}\right)$ will be $C^{\infty}$ throughout the region where $H$ is defined (including those points where $\sigma=0$ ). Thus, $H$ satisfies all the requirements of the argument given above. Hence, we have proven the following "local version" of our theorem.

Lemma 1. Let $G\left(x, x^{\prime}\right)$ satisfy the wave equation in each variable and be Hadamard in an open neighborhood $\mathcal{O}$ of a Cauchy surface $S$. Let $\mathscr{U}$ be an open set such that $H$ is defined for all $x, x^{\prime} \in \mathscr{U}$ and such that the Cauchy development within $\mathscr{U}$ of $\mathscr{U} \cap S$ contains all of $\mathscr{U}$ (see Fig. 2). Then $G$ is Hadamard throughout $\mathscr{U}$ to the future of $S$.

1 Symmetry of $u\left(x, x^{\prime}\right)$ and $V\left(x, x^{\prime}\right)=\sum v_{n}\left(x, x^{\prime}\right) \sigma^{n}$ (in the analytic case) follows from the arguments on p. 164 of [10]. We have proven the symmetry of each individual $v_{n}\left(x, x^{\prime}\right)$ by a rather complicated argument involving how these quantities change when a constant parameter ("mass term") is added to the wave operator. At present, however, we have been unable to obtain a simple proof of the symmetry of $v_{n}\left(x, x^{\prime}\right)$ directly from the recursion relations 


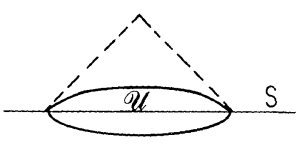

$a$

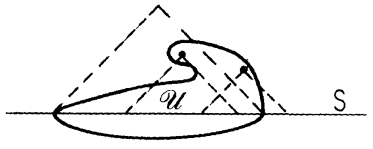

b

Fig. 2 a A set $\mathscr{U}$ with the property required by Lemma 1.b A set which fails to satisfy this property since the two points in $\mathscr{U}$ whose past light cones are shown are not in the Cauchy development within $\mathscr{U}$ of $\mathscr{U} \cap S$

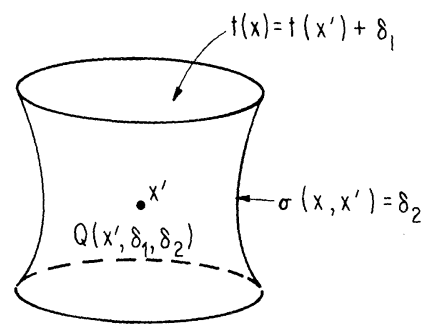

Fig. 3. The set $Q\left(x^{\prime}, \delta_{1}, \delta_{2}\right)$

To obtain the global theorem, we construct a sequence of sets $\mathscr{U}_{j}$ through which we can propagate the Hadamard property by means of Lemma 1. We start by introducing normal neighborhoods for the definition of $H$ which permit a smooth extension of $H$ to larger domains. Let $\left(M, g_{\mu \nu}\right)$ be a globally hyperbolic spacetime with global time function [11] $t$. For each $x^{\prime} \in M$ and numbers $\delta_{1}, \delta_{2}>0$ we define $\tilde{Q}\left(x^{\prime}, \delta_{1}, \delta_{2}\right)$ to be the set of points $x \in M$ with $\left|t(x)-t\left(x^{\prime}\right)\right|<\delta_{1}$ such that there is at least one geodesic connecting $x^{\prime}$ and $x$ with $\sigma<\delta_{2}$. To avoid potential pathologies, we define $Q\left(x^{\prime}, \delta_{1}, \delta_{2}\right)$ to be the connected component of $\tilde{Q}$ containing $x^{\prime}$. The set $Q$ is illustrated in Fig. 3. The following lemma enables us to use these sets as normal neighborhoods if we choose $\delta_{1}$ and $\delta_{2}$ sufficiently small.

Lemma 2. Let $K$ be a compact subset of $M$. Then there exist $\delta_{1}>0$ and $\delta_{2}>0$ such that for each $x^{\prime} \in K, Q\left(x^{\prime}, \delta_{1}, \delta_{2}\right)$ is a normal neighborhood of $x^{\prime}$.

Outline of Proof. Assume the contrary. Then we can find a sequence of points $x_{n}^{\prime}$ such that $Q\left(x_{n}^{\prime}, \delta_{1}, \delta_{2}\right)$ is not a normal neighborhood. Since $K$ is compact, we can find a subsequence $\left\{y_{n}\right\}$ of $\left\{x_{n}^{\prime}\right\}$ which converges to a point $y \in K$. Let $\mathscr{N}$ be a convex normal neighborhood of $y$. By straightforward but tedious arguments, one can show that for sufficiently large $N$, the set $Q\left(y_{N}, \frac{1}{N}, \frac{1}{N}\right)$ is entirely contained within $\mathscr{N}$. (To prove this we first show that we can find $\gamma_{1}, \gamma_{2}>0$ such that $Q\left(y, \gamma_{1}, \gamma_{2}\right)$ is entirely contained within $\mathscr{N}$ and then show that for all $z$ in a certain neighborhood of $y$, and for all $\lambda_{1}<\frac{\gamma_{1}}{2}, \lambda_{2}<\frac{\gamma_{2}}{2}$ we have $Q\left(z, \lambda_{1}, \lambda_{2}\right) \subset Q\left(y, \gamma_{1}, \gamma_{2}\right)$.) This contradicts the assumption that $Q\left(y_{N}, \frac{1}{N}, \frac{1}{N}\right)$ is not normal, thus proving the lemma.

The complete proof of our theorem can now be given. 


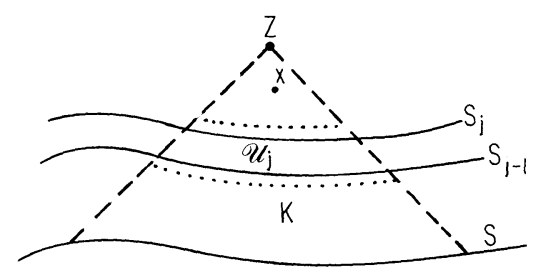

Fig. 4. The set $\mathscr{U}_{j}$

Proof of Theorem. Let $x \in M$, say $x \in D^{+}(S)$ where $D^{+}(S)$ denotes the future domain of dependence of $S$. We will show that $G$ is Hadamard in a neighborhood of $x$. Let $z$ lie to the future of $x$ and let $K=J^{-}(z) \cap D^{+}(S)$, where $J^{-}(z)$ denotes the causal past of $z$. Then $K$ is compact [11]. Let $t$ be a global time function on $M$ with the surface $t=0$ coinciding with $S$. For this choice of $K$ and $t$, let $\delta_{1}$ and $\delta_{2}$ be as in Lemma 2. Let $N$ be an integer with $N \delta_{1}>2 t(z)$. Let $t_{j}=t(z) j / N$ and let $S_{j}$ be the Cauchy surface $t=t_{j}$. Let $\mathscr{U}_{j}=\mathscr{V}_{j} \cap \operatorname{int}(K)$ where $\mathscr{V}_{j}$ consists of the points $w$ such that $t_{j-1}-\frac{\delta_{1}}{4}<t(w)<t_{j}+\frac{\delta_{1}}{4}$. The set $\mathscr{U}_{j}$ is illustrated in Fig. 4. Choose $\beta$ and $\alpha_{n}$ so that $\max \left(\beta, \alpha_{n}\right)<\delta_{2}$. For $x$ and $x^{\prime}$ in $\mathscr{U}_{j}$, define $H_{j}\left(x, x^{\prime}\right)$ by (8) if $x \in Q\left(x^{\prime}, \delta_{1}, \delta_{2}\right)$ and by $H_{j}\left(x, x^{\prime}\right)=0$ otherwise. Then, for $j \geqq 1$, the quantities $\left(S_{j}, \mathscr{U}_{j}, \mathscr{U}_{j+1}, H_{j+1}\right)$ satisfy the conditions required of $(S, \mathcal{O}, U, H)$ in Lemma 1 . Thus, by Lemma 1 , if $G$ is Hadamard in $U_{j}$ it is also Hadamard throughout $\mathscr{U}_{j+1}$. But Lemma 1 also implies that since $G$ is Hadamard in the open neighborhood $\mathcal{O}$ of $S=S_{0}$, it is Hadamard throughout $\mathscr{U}_{1}$. Thus, by induction, $G$ is Hadamard throughout every $\mathscr{U}_{j}$, i.e., throughout int $(K)$, which is an open neighborhood of the (arbitrary) point $x$. This completes the proof.

\section{References}

1. Christensen, S.M.: Vacuum expectation value of the stress tensor in an arbitrary curved background: The covariant point separation method. Phys. Rev. D 14, 2490 (1976)

2. Christensen, S.M.: Regularization, renormalization, and covariant geodesic point separation. Phys. Rev. D 17, 946 (1978)

3. Davies, P.C.W., Fulling, S.A., Christensen, S.M., Bunch, T.S.: Energy-momentum tensor of a massless scalar quantum field in a Robertson-Walker universe. Ann. Phys. (N. Y.) 109, 108 (1977)

4. Bunch, T.S., Christensen, S. M., Fulling, S.A.: Massive quantum field theory in two-dimensional Robertson-Walker spacetimes. Phys. Rev. D (in press)

5. Adler, S.L., Lieberman, J., Ng, Y.J.: Regularization of the stress-energy tensor for vectors and scalar particles propagating in a general background metric. Ann. Phys. (N.Y.) 106, 279 (1977)

6. Wald, R.M.: The back reaction effect in particle creation in curved spacetime. Commun. math. Phys. 54, 1 (1977)

7. Wald, R.M.: Trace anomaly of a conformally invariant quantum field in curved spacetime. Phys. Rev. D 17, 1477 (1978)

8. Hadamard, J.: Lectures on Cauchy's problem in linear partial differential equations. New Haven: Yale University Press 1923

9. DeWitt, B.S., Brehme, R.W.: Radiation damping in a gravitational field. Ann. Phys. (N.Y.) 9, 220 (1960)

10. Garabedian, P.R. : Partial differential equations. New York: Wiley 1964 
11. Hawking, S.W., Ellis, G.F.R.: The large scale structure of the universe. Cambridge: University Press 1973

12. Bjorken, J.D., Drell,S.D. : Relativistic quantum fields, Appendix C. New York : McGraw-Hill 1965

13. Ford, L., Parker, L.: Infrared divergences in a class of Robertson-Walker universes. Phys. Rev. D 16, 245 (1977)

14. Powers, R.T.: Self-adjoint algebras of unbounded operators. Commun. math. Phys. 21, 85 (1971), Section VI

Communicated by R. Geroch

Received June 16, 1978 